\title{
The very massive star content of the nuclear star clusters in NGC 5253
}

\author{
Linda J. Smith ${ }^{1}$, Paul A. Crowther ${ }^{2}$ and Daniela Calzetti ${ }^{3}$ \\ ${ }^{1}$ European Space Agency and Space Telescope Science Institute, \\ 3700 San Martin Drive, Baltimore, MD 21218, USA \\ email: 1smith@stsci.edu \\ ${ }^{2}$ Dept. of Physics and Astronomy, University of Sheffield, Sheffield S3 7RH, UK \\ email: paul.crowther@sheffield.ac.uk \\ ${ }^{3}$ Dept. of Astronomy, University of Massachusetts - Amherst, Amherst, MA 01003, USA \\ email: calzetti@astro.umass.edu
}

\begin{abstract}
The blue compact dwarf galaxy NGC 5253 hosts a very young starburst containing twin nuclear star clusters. Calzetti et al. (2015) find that the two clusters have an age of $1 \mathrm{Myr}$, in contradiction to the age of 3-5 Myr inferred from the presence of Wolf-Rayet (W-R) spectral features. We use Hubble Space Telescope (HST) far-ultraviolet (FUV) and ground-based optical spectra to show that the cluster stellar features arise from very massive stars (VMS), with masses greater than $100 \mathrm{M}_{\odot}$, at an age of 1-2 Myr. We discuss the implications of this and show that the very high ionizing flux can only be explained by VMS. We further discuss our findings in the context of VMS contributing to He II $\lambda 1640$ emission in high redshift galaxies, and emphasize that population synthesis models with upper mass cut-offs greater than $100 \mathrm{M}_{\odot}$ are crucial for future studies of young massive clusters.
\end{abstract}

Keywords. galaxies: dwarf - galaxies: individual (NGC 5253) - galaxies: starburst - galaxies: star clusters: general - stars: massive - stars: Wolf-Rayet

\section{Introduction}

NGC 5253 is a blue compact dwarf galaxy at a distance of $3.15 \mathrm{Mpc}$ (Freedman et al. 2001, Davidge 2007). It hosts a very young central starburst; the near-absence of nonthermal radio emission suggests that very few supernovae have exploded so far (Beck et al. 1996). The galaxy contains many young massive clusters, which display W-R emission features, suggesting that the burst is 3-5 Myr old (Campbell et al. 1986, Schaerer et al. 1997). The metallicity of NGC 5253 is $35 \%$ solar (Monreal-Ibero et al. 2012). Observations at radio wavelengths show that the central region is dominated by a massive ultracompact H II region (Turner et al. 2000). Alonso-Herrero et al. (2004) used the Near Infrared Camera and Multi Object Spectrometer (NICMOS) on HST to discover a double nuclear star cluster with the two components separated by $0^{\prime \prime} .3-0 .{ }^{\prime \prime} 4(\approx 5 \mathrm{pc})$. The eastern cluster coincides with the peak of the $\mathrm{H} \alpha$ emission in NGC 5253 and is the youngest optical star cluster identified by Calzetti et al. (1997). The western cluster is very reddened and is coincident with the ultracompact H II region (Turner et al. 2000).

Recently, Calzetti et al. (2015) presented a detailed analysis of the two nuclear clusters (\#5 and \#11 in their terminology) using HST imaging from the FUV to the IR. By fitting their spectral energy distributions (SED), they find that the two clusters are extremely young and massive with ages of only $1 \pm 1 \mathrm{Myr}$ and masses of $7.5 \times 10^{4}$ and $2.5 \times 10^{5} \mathrm{M}_{\odot}$ for \#5 and \#11 respectively. In Fig. 1, we show the central region of NGC 5253 containing the clusters \#5 and \#11, taken from Calzetti et al. (2015). The young ages contradict the 


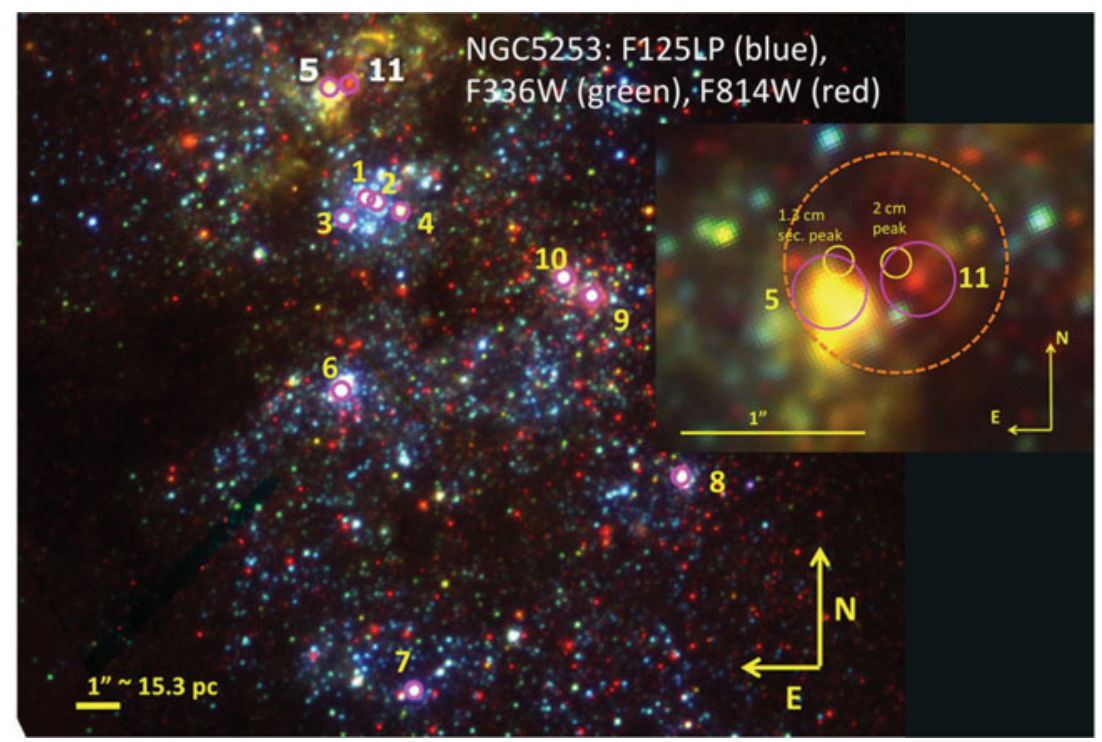

Figure 1. Three-color composite of the central $20^{\prime \prime} \times 16^{\prime \prime}(300 \times 250 \mathrm{pc})$ of NGC 5253 from Calzetti et al. (2015). The 11 brightest clusters from this study are identified with circles and numbered. The inset shows a detailed view of the two nuclear clusters \#5 and \#11, which are separated by a projected distance of $5 \mathrm{pc}$. The small circles correspond to the peaks of the radio emission (Turner et al. 2000). The absolute astrometric uncertainty of the HST images is comparable to the radius of the larger circles $\left(0^{\prime \prime} .2\right)$.

values of 3-5 Myr inferred from the presence of W-R stars in cluster \#5 (e.g. MonrealIbero et al. 2010). We consider here whether the $\mathrm{W}-\mathrm{R}$ features could instead arise from hydrogen-rich very massive stars (VMS; masses $>100 \mathrm{M}_{\odot}$ ). The full study is described in Smith et al. (2016).

In a series of papers studying the core of the cluster R136 in the 30 Doradus region of the Large Magellanic Cloud, Crowther et al. (2010, 2016) used FUV spectra obtained with spectrographs on HST to determine the masses of the most massive stars using modelling techniques. They found that the R136 cluster is only $1.5 \pm 0.5 \mathrm{Myr}$ old and contains eight stars more massive than $100 \mathrm{M}_{\odot}$, with the most massive star (called R136a1) having a current mass of $315 \pm 50 \mathrm{M}_{\odot}$. The four most massive stars account for one-quarter of the total ionizing flux from the star cluster. These VMS have very dense, optically thick winds and their emission-line spectra resemble W-R stars, but they are hydrogen-rich and on the main sequence.

\section{Observations and Results}

We obtained FUV Space Telescope Imaging Spectrograph (STIS) and Faint Object Spectrograph (FOS) spectra of cluster \#5 from the Mikulski Archive for Space Telescopes (MAST). The STIS G140L spectrum covers 1150-1730 $\AA$ with a spectral resolution of $1.8 \AA$. The FOS G190H spectrum has a resolution of $3.8 \AA$ and covers $1590-2310 \AA$. The two spectra were merged in the overlap region.

In Fig. 2, we compare the HST/STIS+FOS spectrum of cluster \#5 with the integrated HST/STIS spectrum of R136a from Crowther et al. (2016). The similarity between the two spectra is striking. The strengths, widths and velocities of the $\mathrm{N} \mathrm{v}, \mathrm{O}$ v and $\mathrm{C}$ IV lines are in excellent agreement between the two spectra. The crucial VMS spectral features are the presence of blue-shifted O v $\lambda 1371$ wind absorption, broad He II $\lambda 1640$ emission, and 


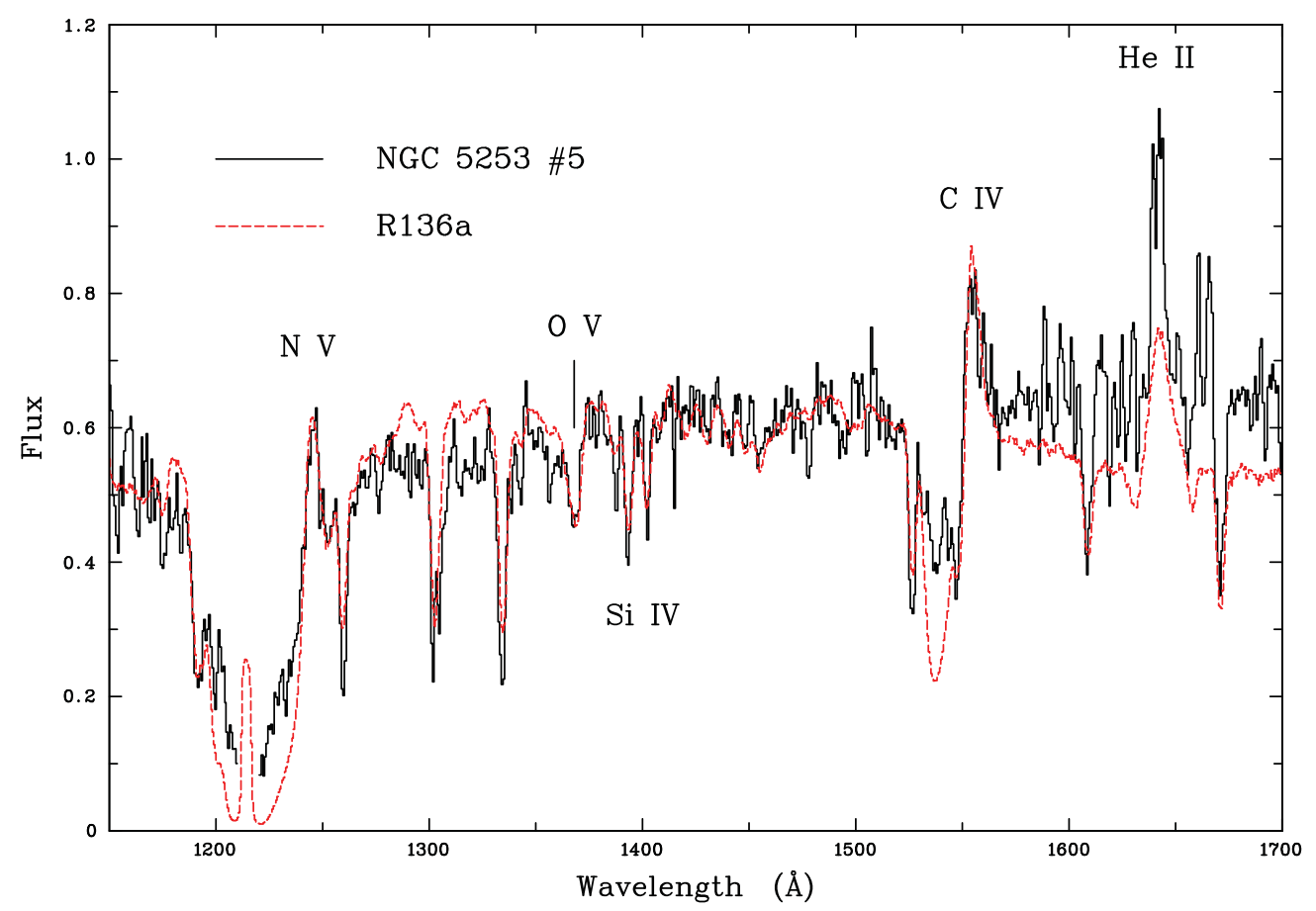

Figure 2. Integrated HST/STIS spectrum of R136a from Crowther et al. (2016) compared to the HST/STIS+FOS spectrum of cluster \#5. The R136a spectrum has been scaled to the distance of NGC 5253. The flux is in units of $10^{-15} \mathrm{ergs} \mathrm{cm}^{-2} \mathrm{~s}^{-1} \AA^{-1}$.

the absence of a Si IV $\lambda 1400$ P Cygni profile (expected in classical W-R stars). Crowther et al. (2016) find that $95 \%$ of the broad He II emission shown in the R136a spectrum in Fig. 2 originates solely from VMS. Thus, the presence of this feature in emission together with the $\mathrm{OV}$ wind absorption indicates a very young age $(<2 \mathrm{Myr})$ for cluster \#5 and a mass function that extends well beyond $100 \mathrm{M}_{\odot}$.

We have also obtained high spectral resolution spectroscopy across the starburst core of NGC 5253 with the UV-Visual Echelle Spectrograph (UVES) at the Very Large Telescope (VLT). This spectrum covers 3100-10360 $\AA$ and thus both the blue and red W-R bumps. The red bump due to WC C IV $\lambda \lambda 5801,5812$ emission is absent. The blue bump corresponds to the W-R emission features of N III $\lambda 3634-4641$, C III $\lambda 4647-4651$ and He II $\lambda 4686$. We show this region in Fig. 3. Broad He II emission $\left(1450 \mathrm{~km} \mathrm{~s}^{-1}\right)$ is the only $\mathrm{W}$-R feature present. N III is present but narrow and the individual line components are resolved. They are likely to be nebular in origin, although detecting these transitions is unusual. We also detect nebular He II $\lambda 4686$. The blue bump region of the VMS in R136 is dominated by strong broad He II $\lambda 4686$ with N III absent (Caballero-Nieves et al. in prep.). From this, the optical signature of VMS is strong and broad He II $\lambda 4686$ emission and N III $\lambda 3634-4641$ absent.

\section{Discussion and Conclusions}

We have examined UV and optical spectroscopy of cluster \#5 in the nucleus of NGC 5253 with the aim of reconciling the extremely young age of $1 \pm 1 \mathrm{Myr}$ found by Calzetti et al. (2015) with the presence of W-R features in the cluster spectrum. Specifically, we have investigated whether the $\mathrm{W}$ - $\mathrm{R}$ features arise from hydrogen rich, very massive stars 


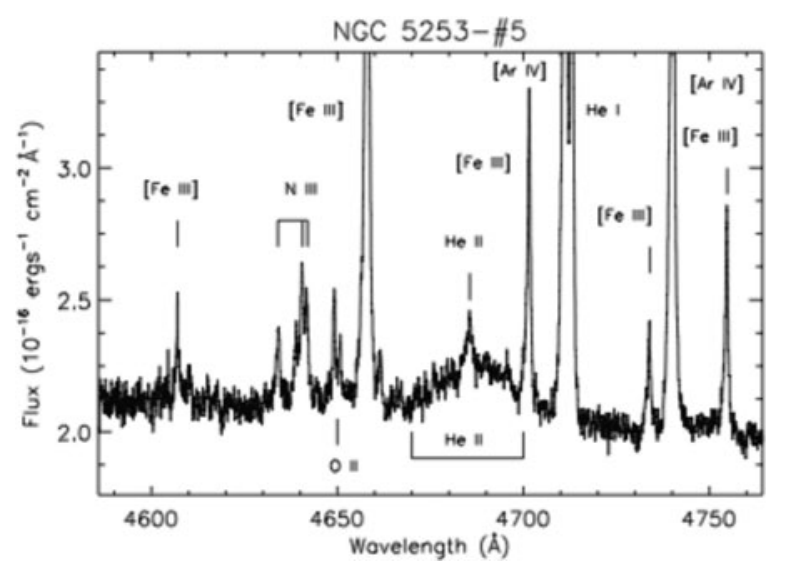

Figure 3. VLT/UVES spectrum of NGC 5253-cluster \#5 in the region of the W-R blue bump.

with masses greater than $100 \mathrm{M}_{\odot}$. We conclude from the UV and optical comparisons with R136 that cluster \#5 is very young with an age of less than 2 Myr and that the broad He II emission is produced by VMS that have dense, optically thick winds and show W-R-like emission features while they are on the main sequence.

The presence of VMS in the nuclear region of NGC 5253 can explain the very high ionizing flux of $Q(\mathrm{H} \mathrm{I})=2.2 \times 10^{52} \mathrm{~s}^{-1}$ for the central $5 \mathrm{pc}$ region (Turner \& Beck 2004). Previous studies (e.g. Turner et al. 2015) have assumed an age of 3-5 Myr and found that the predicted ionizing fluxes from Starburst99 (Leitherer et al. 1999) significantly underpredict the ionizing flux, unless a top heavy initial mass function is assumed. Calzetti et al. (2015) likewise find that $50 \%$ of the ionizing flux is unaccounted for using a lower age of 1 Myr and SED modelling with an upper mass cut-off of $100 \mathrm{M}_{\odot}$. For R136, Doran et al. (2013) find that $Q(\mathrm{HI})=7.5 \times 10^{51} \mathrm{~s}^{-1}$ and that the four most massive VMS produce $25 \%$ of this ionizing flux. We find that the extra ionizing flux needed for the central 5 pc of NGC 5253 at an age of 2 Myr over Starburst99 (with an upper mass cutoff of $100 \mathrm{M}_{\odot}$ ) is the equivalent of $12 \mathrm{VMS}$. This number seems reasonable given the six times greater mass for clusters \#5 and \#11 compared to R136.

The FUV spectrum of cluster \#5 is representative of a very young, nearby, low metallicity, nuclear starburst. It bears a striking resemblance to the UV rest-frame spectrum of Q2343-BX418 (Erb et al. 2010). These authors suggest this $z=2.3$ young, low mass, low metallicity galaxy is a plausible analogue to the young, low metallicity star-forming galaxies at $z>5$. Q2343-BX418 has broad He II $\lambda 1640$ emission, and nebular emission from O III] $\lambda \lambda 1661,1666$ and C III] $\lambda \lambda 1907,1909$. The $\mathrm{C}$ III] equivalent width of $7.1 \AA$ is very similar to the value of $7.7 \AA$ that we measure for the FOS spectrum near cluster $\# 5$.

The James Webb Space Telescope (JWST) will obtain rest frame UV spectra of high redshift star-forming galaxies. These spectra may reveal the presence of VMS through the presence of broad He II $\lambda 1640$ emission and OV $\lambda 1371$ wind absorption, and the absence of Si IV $\lambda 1400$ P Cygni emission. Population synthesis models typically have upper mass cutoffs of $100 \mathrm{M}_{\odot}$. For all studies near and far, it is crucial to extend these into the VMS regime to correctly account for the radiative, mechanical and chemical feedback, which will be dominated by VMS for the first 1-3 Myr in star-forming regions. 


\section{References}

Alonso-Herrero, A., Takagi, T., Baker, A. J., et al. 2004, ApJ, 612, 222

Beck, S. C., Turner, J. L., Ho, P. T. P., Lacy, J. H., \& Kelly, D. M. 1996, ApJ, 457, 610

Calzetti, D., Meurer, G. R., Bohlin, R. C., et al. 1997, AJ, 114, 1834

Calzetti, D., Johnson, K. E., Adamo, A., et al. 2015, ApJ, 811, 75

Campbell, A., Terlevich, R., \& Melnick, J. 1986, MNRAS, 223, 811

Crowther, P. A., Schnurr, O., Hirschi, R., et al. 2010, MNRAS, 408, 731

Crowther, P. A., Caballero-Nieves, S. M., Bostroem, K. A., et al. 2016, MNRAS, 458, 624

Davidge, T. J. 2007, AJ, 134, 1799

Doran, E. I., Crowther, P. A., de Koter, A., et al.2013, A\&A A, 558, A134

Erb, D. K., Pettini, M., Shapley, A. E., et al. 2010, ApJ, 719, 1168

Freedman, W. L., Madore, B. F., Gibson, B. K., et al. 2001, ApJ, 553, 47

Leitherer, C., Schaerer, D., Goldader, J. D., et al. 1999, ApJS, 123, 3

Monreal-Ibero, A., Vílchez, J. M., Walsh, J. R., \& Muñoz-Tuñón, C. 2010, A\&̇, 517, A27

Monreal-Ibero, A., Walsh, J. R., \& Vílchez, J. M. 2012, A\&SA, 544, A60

Schaerer, D., Contini, T., Kunth, D., \& Meynet, G. 1997, ApJ, 481, L75

Smith, L. J., Crowther, P. A., Calzetti, D., \& Sidoli, F. 2016, ApJ, 823:38

Turner, J. L., Beck, S. C., Benford, D. J., et al. 2015, Nature, 519, 331

Turner, J. L. \& Beck, S. C. 2004, ApJ, 602, L85

Turner, J. L., Beck, S. C., \& Ho, P. T. P. 2000, ApJ, 532, L109 